\title{
Produção de vídeo-aulas como ferramenta de aprendizagem para a disciplina de anatomia animal
}

The production of video-classes as a learning tool to the animal anatomy discipline

\author{
Luciana Silveira Flores Schoenau', William Schoenau²
}

\author{
I Professora de Anatomia Animal do Departamento de Morfologia, Centro de Ciências da Saúde/UFSM. Doutora em Anatomia Animal. \\ Ischoenau@gmail.com
}

2 Professor de Fisiologia do Departamento de Fisiologia e Farmacologia do Centro de Ciências da Saúde/UFSM. Doutor em Medicina Veterinária.wschoenau@smail.ufsm.br

\section{Resumo}

O estudo da anatomia tem sido um desafio nos últimos tempos. A utilização de animais no ensino tem sido dificultada por questões éticas e morais, pela corrente dos $3 \mathrm{R}$ (reduzir, reciclar e reutilizar), o que dificulta desta forma a aquisição das peças e a manipulação das mesmas pelos estudantes. Isso afetou diretamente a metodologia mais tradicional e eficaz de aprendizagem da anatomia: a dissecção. Aliado a esses fatores, não se pode negar que os estudantes ingressantes no ensino superior fazem parte da geração tecnológica, passam grande parte do seu tempo em frente a um computador e utilizam equipamentos digitais. A busca de novas tecnologias no ensino da anatomia é uma realidade atualmente. Este trabalho teve como objetivo produzir uma ferramenta de aprendizagem através do vídeo e de avaliar a sua utilização pelos acadêmicos do Curso de Medicina Veterinária. Foi proposta a elaboração de vídeos de determinados assuntos para grupos de no mínimo três estudantes como parte da avaliação da disciplina. Os resultados foram analisados de forma qualitativa e quantitativa, através de um formulário baseado na escala de Likert de cinco pontos. Os resultados mostraram-se favoráveis a utilização do vídeo como ferramenta de aprendizagem e como fator de integração do grupo.

Palavras Chave: anatomia, ensino, vídeo, aprendizagem.

\begin{abstract}
The anatomy study has been a challenge lately. The use of animals in education has been a difficult due by moral and ethical issues and the 3R's chain (reduce, recycle and reuse), which makes it difficult the acquisition of anatomical parts and the manipulation of them by the students. This issue affected directly the more traditional and effective methodology of learning anatomy: the dissection. Allied to these factors, the students of higher education belong to a technological generation, spending much of their time in front of the computer and using digital equipment. The search of new technologies of anatomical education is now a reality. The aim of this research was to produce a learning tool through video and to evaluate its use by students of Veterinary Medicine. The video production of different issues was proposed to groups of at least three students as part of the evaluation of the subject. The students have answered a survey based on a five-point Likert scale and the results have been analyzed by qualitative and quantitative ways. The results have been favorable for the use of video as a learning tool and a way of group integration.
\end{abstract}

Keywords: anatomy, education, video, learning. 


\section{INTRODUÇÃO}

A anatomia é, sem sombra de dúvida, uma das disciplinas mais importantes do curso de fundamentação médica e de importância indiscutível para a área biomédica. É uma disciplina difícil, pois na maioria das vezes requer a memorização de nomes de muitas estruturas. Sua essência é o aprendizado através da visualização, seja através de aulas práticas demonstrativas, dissecação de cadáveres ou ainda através de imagens. É uma ciência que só coexiste com a prática.

A busca da preservação das espécies e do fim do sacrifício dos animais para o bem da ciência constituem paradigmas para o futuro da anatomia, o que torna muito grande a busca de alternativas que deixem essa disciplina mais atrativa e produtiva. Há uma transição do uso de cadáveres para a entrada de novas tecnologias como uma forma de complementação dos acessos e recursos tradicionais e problemas éticos (KINISSON et al., 2009).

Tradicionalmente, a anatomia é estudada por profissionais e/ou estudantes da área de três maneiras distintas e individuais: textos, atlas e cadáveres. Através dos textos o leitor tenta "imaginar" as complexas relações anatômicas. $\mathrm{O}$ atlas apresenta o conteúdo na forma de desenhos esquemáticos ou fotografias de peças anatômicas reais, ficando o usuário limitado às ilustrações apresentadas pelo autor, não tendo acesso à representação de todos os ângulos possíveis e desejáveis ao estudo (INFANTOSI; KLEMT, 2000).

O recurso instrucional pode ser definido como qualquer elemento utilizado nos procedimentos de ensino para estimular a percepção e atenção do aluno. Complementa a ação do instrutor, sendo um componente importante no ambiente de aprendizagem. Atualmente, um orientador não deve simplesmente transmitir seus conhecimentos ou informações, mas também apontar aos seus discentes o modo de aprender e buscar o prazer do descobrir. Por isso, o simples ato de aprender deve ser o mais agradável e amigável possível, ainda que a aquisição de conhecimentos sempre exija esforços e dedicação (YOSHIDA et al., 2003).

O uso de recursos multimídia pode auxiliar a atender esse objetivo, por ser um elemento complementar dentro da aula e por permitir que o aluno reveja rápida e repetidamente os conhecimentos já adquiridos (ALMEIDA, 2009). O recurso multimídia pode apoiar o método de aprendizagem em nível básico, ajudando na percepção visual através da informação de diferentes maneiras usando formas, gráficos, cores e movimento; e aprendizado interativo que pode ser obtido através de jogos estratégicos, criação de situações e interação com outros usuários (DAVIS, 1996).

A produção de vídeo-aula tem sido utilizada como ferramenta complementar de aprendizagem no ensino fundamental (ALMEIDA et al., 2009 e SILVA et al., 2010) e da anatomia humana no ensino da graduação (VERRI et al., 2011)

O vídeo carrega características de recurso pedagógico e é capaz de desenvolver atitudes perceptivas múltiplas nos estudantes. $\mathrm{Na}$ aprendizagem, é frequentemente destacado por suas funções lúdica, motivadora, informativa, avaliativa, conceitual, documental, metalinguística e procedimental (CORREIA; CHAMBEL, 2004).

$O$ vídeo deve ser utilizado não somente para reforçar o que foi ensinado pelo professor em sala de aula, mas para ativar os sentidos dos alunos, sua crítica e exemplificar de forma mais abrangente, facilitando todo o processo de ensino aprendizagem. A utilização de vídeos para o ensino da anatomia humana, mesmo aqueles de produção amadora, contribuem para alcançar satisfatoriamente, os objetivos propostos, o que melhora significativamente a compreensão dos alunos sobre o conteúdo anatômico abordado (SILVA; SANTANA, 2012).

Os vídeos de dissecações, laparoscopias, autópsias, procedimentos clínicos e cirurgias em geral têm sido cada vez mais utilizados para complementar o aprendizado prático de laboratório. As vantagens são muitas: as lentes oferecem visões in vivo e em grande aumento e todos os estudantes recebem a mesma informação em um curto espaço de tempo, (FITZPATRICK et al, 2001).

A vídeo-aula pode ser um estímulo ao estudo e à conscientização sobre a importância da anatomia na formação profissional dos discentes da área da Saúde. Pode-se, ainda, acrescentar que, para as Instituições que estão em pleno investimento na $\mathrm{EAD}$, materiais dessa natureza são meios interativos que criam uma interface entre o aluno e o computador com melhoria na qualidade de ensino (VERRI et al, 2011).

Considerando o atual cenário da Educação à distância no Brasil que se encontra em processo de rápida ascensão, principalmente devido ao aumento da demanda por educação continuada e atualização profissional, faz-se necessário a produção de material didático especializado que possa estar disponível para utilização em ambientes virtuais. A plataforma virtual de ensino e aprendizagem é atualmente uma realidade e sua potencialidade no ensino da anatomia depende em grande parte do fornecimento de materiais audiovisuais, porque essa área de conhecimento só é compreendida 
através da visualização e o vídeo é o meio audiovisual que mais se aproxima da realidade.

Esta pesquisa teve como objetivos o desenvolvimento pelos acadêmicos de uma ferramenta de aprendizagem por meio de imagens para o ensino da anatomia animal no ensino superior, através da elaboração de um vídeo associado a imagens fotográficas e textos de diferentes sistemas corporais para o conhecimento dos diferentes órgãos, elementos anatômicos e de suas relações em diferentes ângulos de visão; viabilizar o uso de vídeos no ensino superior, para que se constitua em uma ferramenta didática que facilite e motive o processo ensino/aprendizagem e avaliar o uso do vídeo como trabalho para a disciplina de anatomia animal.

\section{METODOLOGIA}

A pesquisa foi desenvolvida com acadêmicos do terceiro semestre do Curso de Medicina Veterinária como um trabalho prático para a Disciplina de Anatomia dos Animais Domésticos C. Todos os trabalhos foram realizados no Setor de Anatomia Animal do Departamento de Morfologia do Centro de Ciências da Saúde.

Os temas foram sorteados e distribuídos em grupos de três alunos, para que desta forma todos se envolvessem na elaboração do trabalho. A definição de um número mínimo baseou-se na necessidade de funções básicas e necessárias de narração, filmagem e demonstração. Foram definidos temas curtos para produção do video com um tempo máximo de 20 minutos. Os temas sorteados foram: anatomia da cavidade bucal, palato mole e faringe; anatomia da laringe; anatomia das vias aéreas inferiores; anatomia do ovário e tuba uterina, e anatomia do testículo e escroto. Foram desenvolvidos 15 vídeos, três para cada tema, considerando que as turmas estavam divididas em três grupos. À medida que os trabalhos eram finalizados, stes foram disponibilizados na plataforma virtual Moodle da disciplina.

As peças utilizadas para a produção do video foram peças do acervo laboratorial e disponibilizadas pelo Setor de Anatomia Animal e peças frescas doadas ou obtidas de matadouros pelos próprios acadêmicos.

Para execução da filmagem foram disponibilizados aos acadêmicos uma filmadora Sony Handycam DCR-SR68, um tripé, mesa e campo de fundo. Previamente, os acadêmicos participaram de uma palestra teórico-prática no Núcleo Setorial de Informática (NUSI) do Centro de Ciências Rurais sobre a elaboração de vídeo no Programa Windows Movie Maker.

Para realização do vídeo, foi necessário que o grupo elaborasse um roteiro de pré-produção do tema a ser abordado com produção de texto na forma escrita, selecionasse as peças adequadas para viabilização da sequência do roteiro (discussão com o grupo), identificassem os elementos importantes, executassem a filmagem em várias etapas e finalmente editassem o vídeo com introdução, desenvolvimento e créditos. Em alguns casos foi preciso refazer os vídeos várias vezes.

Os resultados foram avaliados através de um formulário impresso baseado na escala de Likert em 5 pontos $\mathrm{O}$ instrumento de avaliação pedagógica foi composto por 16 itens distribuídos em três critérios: satisfação pessoal e da realização em grupo (6 itens), contribuição do vídeo (7 itens), e uso das tecnologias ( 3 itens). Cada item possuía cinco categorias de respostas, em uma escala tipo Likert: (1) discordo totalmente, (2) discordo parcialmente, (3) sem opinião formada, (4) concordo parcialmente e (5) concordo totalmente. No formulário oi deixado espaço para comentários (prós e contras) sobre a elaboração do vídeo. O formulário foi respondido de forma voluntária e anônima após a entrega do trabalho do vídeo.

A avaliação visou detectar o grau de satisfação, motivação e dificuldades dos participantes na elaboração do vídeo.

Os dados coletados pela aplicação do instrumento de avaliação foram analisados pela média aritmética simples das pontuações obtidas nos itens dos três critérios. De uma turma de 47 estudantes, 35 responderam ao questionário, sendo 11 do sexo masculino e 24 do sexo feminino. Dos 35 acadêmicos ,13 deixaram considerações e desses 7 mulheres e 6 homens.

\section{RESULTADOS E DISCUSSÃO}

O critério satisfação pessoal e de realização em grupo (Tabela 1) obteve média geral de 4,22. A maioria dos itens deste critério teve avaliações positivas, ou seja, apresentaram médias superiores a quatro (concordo parcialmente). De acordo com Caetano e Falkenbach (2007), a democratização do vídeo é uma realidade. As formas de captação baratearam muito, permitindo que qualquer pessoa com um celular ou uma câmera fotográfica digital, consiga fazer vídeo. E os vídeos resultantes podem ser exibidos na internet em um arquivo do 
Tabela 1 - Critério de avaliação de satisfação pessoal e da realização em grupo.

\begin{tabular}{|c|c|c|c|}
\hline \multirow[t]{2}{*}{ SATISFAÇÃO PESSOAL E DA REALIZAÇÃO EM GRUPO } & \multicolumn{3}{|c|}{ Média } \\
\hline & $\begin{array}{c}\hat{\sigma} \\
(n=11)\end{array}$ & $\begin{array}{c}q \\
(n=24)\end{array}$ & $\begin{array}{c}\text { 우 } \\
(\mathrm{n}=35)\end{array}$ \\
\hline 1. Senti-me estimulado com a realização do vídeo & 4,54 & 4,50 & 4,51 \\
\hline 2. A confecção do vídeo é de fácil elaboração. & 4,27 & 3,00 & 3,40 \\
\hline 3. Fiquei satisfeito com o resultado & 4,63 & 4,50 & 4,54 \\
\hline 4. O trabalho propiciou a integração dos componentes do grupo & 4,90 & 4,83 & 4,85 \\
\hline 5. A proposta de realização do vídeo estimulou a liderança e a organização & 4,45 & 4,54 & 4,51 \\
\hline 6. Trabalhos como esse não me sobrecarregam & 4,10 & 3,29 & 3,52 \\
\hline Média final & 4,48 & 4,11 & 4,22 \\
\hline
\end{tabular}

Microsoft PowerPoint ${ }^{\circledR}$, em um computador, em uma TV, etc. $\mathrm{O}$ vídeo permeia uma associação entre o entretenimento e o lazer. Para Moran (2005), esse é um fator importante, pois ao se usar o vídeo em sala de aula o aluno associa-o ao prazer e não à aula.

O critério fortemente indicado (média 4,85) foi de que o trabalho propiciou a integração do grupo. Para os acadêmicos dessa pesquisa, o grau de satisfação com o uso do vídeo foi, acima de tudo, aprender com diversão. Um dos participantes relatou "O trabalho é válido não só para as aulas e provas, mas para a integração e risadas dos erros com os colegas". Além do mais, durante a realização do trabalho com a supervisão do docente responsável pode-se observar o quanto houve integração e divertimento. Outros relatos indicaram que o vídeo estimula a criatividade, a interação com outros meios e propicia a união e a responsabilidade.

Em dois itens, a avaliação foi insatisfatória quando comparada aos demais com média geral abaixo de quatro: a facilidade de elaboração do vídeo $(3,40)$ e a sobrecarga de trabalho $(3,52)$. Estes dois critérios não só se diferenciaram dos demais como houve diferença de sexo. As acadêmicas parecem ter maior dificuldade de elaboração, pois houve uma diferença de 1,27 pontos quando comparados ao sexo masculino bem como se sentem mais sobrecarregadas com diferença de 1,19 pontos. A experiência relatada da produção de vídeo em sala de aula por Silva e Oliveira (2010) com alunos da quinta série desmistificou a concepção de que o processo de produção dos audiovisuais é uma tarefa complexa e impossível de ser realizada em salas de aula. A dificuldade relatada pelos acadêmicos na elaboração do vídeo nessa pesquisa provavelmente se deva a uma maior exigência de alunos no ensino superior. Acredita-se que tal dificuldade esteja aliada a sobrecarga de trabalho relatada pelos acadêmicos já que, além de realizarem trabalho para esta disciplina, eles têm que elaborar outros trabalhos em outras disciplinas do mesmo semestre. Das dificuldades na elaboração do vídeoforam relatadas a falta de climatização na sala de vídeo, qualidade das peças anatômicas, ruídos externos, ausência de panos de fundo extras e acesso a equipamentos de edição de vídeo. Estas observações foram relatadas basicamente pelas acadêmicas $(71,42 \%$ das que fizeram comentários). A única consideração negativa do sexo masculino se limitou ao prazo para entrega do vídeo $(16,66 \%)$. Considerando que atualmente $75,8 \%$ dos ingressantes no Curso de Medicina Veterinária da UFSM são do sexo feminino (CPQMVET, 2012) e provavelmente devido a um maior grau de exigência quanto aos detalhes na realização do vídeo, esses fatores, como sobrecarga de trabalho e dificuldade na realização do vídeo, devem ser levados em consideração e melhor estudados.

De um modo geral, os acadêmicos de ambos os sexos sentiram-se estimulados, satisfeitos com a elaboração e integrados ao grupo, onde houve estímulo à liderança e organização. Uma acadêmica relatou que a realização do vídeo melhora a integração, considerando a necessidade de elaborar um roteiro e selecionar as peças anatômicas. A experiência de elaboração do vídeo em sala de aula é vista como um mecanismo de extrema importância para renovação do contexto escolar seja por mecanismos de inclusão, de democra- 
tização, de ressignificação ou de transformação dos saberes e papéis a serem desempenhados. A promoção de diversos debates induz ao aluno buscar informações (incitando a leitura), possibilita a interdisciplinaridade e promove novas reflexões no contexto escolar e social (SILVA; OLIVEIRA, 2010). A utilização de vídeos na educação auxilia o despertar da curiosidade, permite compor cenários desconhecidos pelos alunos, permite simulações da realidade, reproduz entrevistas, depoimentos, documentários, auxilia no desenvolvimento da construção do conhecimento coletivo pela análise em grupo e o desenvolvimento do senso crítico. $O$ vídeo tem uma dimensão lúdica, pois permite brincar com a realidade e mostrá-la aonde quer o aluno quanto a Instituição. Bravo e Inzunza (1995) demonstraram, por meio de resultados de avaliações, que a associação de programas de computador com os trabalhos práticos melhora o conhecimento teórico dos temas morfológicos.

Davis (1996) citou que a taxa de retenção ao ouvir uma informação é de cerca de $20 \%$, após ver uma informação é de $30 \%$, e ao ver e ouvir uma informação é de 50\%. Enquanto se o indivíduo ouvir, ver e tocar é de $70 \%$. No cérebro, $70 \%$ de todos os receptores, além de $40 \%$ do córtex, são destinados à visão, dado que justifica a interação do aprendizado teórico/prático no processo de ensino obtidos pela estimulação multissensorial. A elaboração do vídeo pelos acadêmicos envolveu

Tabela 2 - Critério de contribuição ao vídeo

\begin{tabular}{|c|c|c|c|}
\hline \multirow[t]{2}{*}{ CONTRIBUIÇÃO DO VÍDEO } & \multicolumn{3}{|c|}{ Média } \\
\hline & $\begin{array}{c}q \\
(n=11)\end{array}$ & $\begin{array}{c}q \\
(n=24)\end{array}$ & $\begin{array}{c}+0^{1} \\
(n=35)\end{array}$ \\
\hline 1. A realização do vídeo traz benefício, não considero uma perda de tempo. & 4,63 & 4,79 & 4,74 \\
\hline 2. A elaboração do vídeo permite a aprendizagem através da experiência & 4,72 & 4,73 & 4,73 \\
\hline 3. O vídeo simula a realidade da peça anatômica & 4,72 & 4,45 & 4,45 \\
\hline 4. Acredito que o vídeo seja uma forma clara de apresentar o conteúdo & 4,80 & 4,70 & 4,74 \\
\hline 5. O uso de tecnologias (vídeo) em aula valoriza o aluno & 4,54 & 4,37 & 4,42 \\
\hline $\begin{array}{l}\text { 6. O uso de tecnologias (vídeo) em aula me estimula a desenvolver } \\
\text { aprendizado extra }\end{array}$ & 4,72 & 4,54 & 4,60 \\
\hline 7. A elaboração de vídeo beneficia tanto o aluno quanto a instituição. & 4,45 & 4,54 & 4,51 \\
\hline Média final & 4,65 & 4,58 & 4,59 \\
\hline
\end{tabular}

que seja necessário ou desejável (MORAN, 2005).

O critério de contribuição do vídeo (Tabela 2) foi indicado positivamente com média de 4,59, não havendo maiores diferenças de sexo. Os itens com maior unanimidade foram aqueles que mostram o vídeo é uma forma clara de apresentar o conteúdo $(4,74)$ se igualando em pontuação a de que este traz benefícios não sendo perda de tempo $(4,74)$ e permite a aprendizagem através da experiência $(4,73)$. Em um comentário, um acadêmico relatou que ao mostrar seu vídeo para um leigo, percebeu que o vídeo facilita a comunicação. Além disso, os acadêmicos acreditam que o uso do vídeo estimula o aprendizado extra e beneficia tanto a visão durante a seleção e demonstração das peças em seus mínimos detalhes, edição do vídeo e finalização. A audição foi explorada durante as discussões com os colegas e leitura do roteiro. Em todos os momentos, as peças precisaram ser manipuladas durante a seleção e demonstração. Além do mais, muitos vídeos tiveram que ser refeitos, reforçando ainda mais o aprendizado. A execução de um trabalho prático baseado no vídeo como instrumento de aprendizagem é multissensorial e, portanto, extremamente favorável ao aprendizado, conforme apontado positivamente pelos acadêmicos nos itens referentes às características do vídeo ser uma forma clara de apresentar o conteúdo $(4,74)$, trazer benefícios $(4,74)$, permitir 
a aprendizagem através da experiência $(4,73)$ e possibilitar um aprendizado extra $(4,6)$. Em uma consideração, uma acadêmica comentou que uma das vantagens do vídeo é poder estudar posteriormente para prova, ou seja, o vídeo se constitui em um reforço de aprendizagem.

Pode-se observar nos comentários que os acadêmicos do sexo masculino apontaram em sua maioria (83\%) a capacidade do vídeo em melhorar o aprendizado, estimulando o estudo e valorizando o aprendizado do aluno. As acadêmicas foram mais detalhistas em seus comentários priorizando suas indicações em relação aos ganhos do grupo (integração, responsabilidade) e dificuldades para realização de um bom vídeo.

Nessa pesquisa, embora com pontuação acima de 4,0, os acadêmicos indicaram, menos positivamente, a afirmação de que o vídeo simularia a realidade da peça anatômica o que parece ir ao encontro com o relatado por Bravo e Inzunza (1995), que dizem ficar claro que o computador não substitui aulas práticas de laboratório, visto que na anatomia a observação e o estudo direto das estruturas tridimensionais são considerados condições fundamentais ao aprendizado.

Salomon (2013) enumerou dez razões para os estudantes criarem vídeos: é divertido; compromete e estimula os estudantes; ajuda a entender o conteúdo; ajuda a reter o que foi aprendido; permite aos alunos expressar a criatividade; chama os alunos para a prática de habilidades da «vida real» de planejamento e organização, gestão do tempo, cooperação, comunicação, resolução de problemas, sintetizar informações e liderança; utiliza habilidades curriculares integrais: ler, pesquisar, escrever, falar e de ouvir; requer que os alunos pensem em um nível mais elevado; leva a uma melhora nas habilidades tecnológicas; e ajuda-os a desenvolver habilidades na resolução de problemas. A criação de vídeos em sala de aula combina um método baseado em projetos de aprendizagem com uma abordagem prática. As tarefas são autênticas e os alunos reforçam as habilidades de criação, colaboração e comunicação do século 21 . O resultado é que os alunos tornam-se participantes ativos em vez de alunos passivos.

A avaliação do uso das tecnologias foi composta de três critérios nos quais os acadêmicos mostraram-se favoráveis com média geral de 4,36. Destes critérios, o melhor pontuado foi o de que os alunos foram favoráveis ao uso de tecnologias em sala de aula. Para medir o interesse natural dos discentes por programas de computador, Bravo \& Inzunza (1995) ofereceram aos alunos a opção de utilizar, de forma absolutamente livre, alguns programas de apoio ao curso de Anatomia. A grande maioria dos estudantes utilizou algum programa, enquanto apenas $21 \%$ não se interessaram. Os programas de multimídia permitiam combinar o uso de textos, figuras de alta qualidade, vídeos de aplicação clínica, som e perguntas de autoavaliação, possibilitando aos estudantes utilizá-los de acordo com seu ritmo particular de aprendizagem. Bravo \& Inzunza (1995) concluíram que a tendência dos alunos em utilizar programas de computador é bastante alta, que o impacto dessa tecnologia no processo de ensino-aprendizagem é significativa, e a aceitação por parte dos alunos é satisfatória, tanto nos aspectos de conteúdos e interação modulo usuário, como na aprendizagem. Com média acima de quatro, mas com indicação menos positiva, ficaram os itens de que os acadêmicos não gostam de ser avaliados somente com provas e de que o trabalho ampliou o conhecimento tecnológico.

Moran (1995) acredita que apesar de todas as oportunidades oferecidas pela tecnologia, o uso do vídeo não adianta nada sem uma boa estrutura pedagógica. Caetano e Falkenbach (2007) acreditam que as tecnologias necessitam de outro elemento que as completem. Alguns autores acham

Tabela 3 - Critério de uso das tecnologias

\begin{tabular}{|c|c|c|c|}
\hline \multirow[t]{2}{*}{ USO DAS TECNOLOGIAS } & \multicolumn{3}{|c|}{ Média } \\
\hline & $\begin{array}{c}0 \\
(n=11)\end{array}$ & $\begin{array}{c}q \\
(\mathrm{n}=24)\end{array}$ & $\begin{array}{c}\text { की } \\
(\mathrm{n}=35)\end{array}$ \\
\hline 1. A realização do trabalho ampliou meus conhecimentos tecnológicos & 4,36 & 4,08 & 4,17 \\
\hline 2. Gosto quando os professores utilizam tecnologias em sala de aula & 4,72 & 4,60 & 4,64 \\
\hline 3. Não gosto de ser avaliado somente com provas & 4,54 & 4,16 & 4,28 \\
\hline Média total & 4,54 & 4,28 & 4,36 \\
\hline
\end{tabular}


que o professor é o elemento da mudança (CARNEIRO, 2001, VALLE; CRUZ, 2003) enquanto Santoro (1997) acredita que tornar o aluno autor ou coautor no processo de criação seria o segredo para tornar o processo mais ativo. Algo que ele participou ou criou, toma uma dimensão muito diferente. E os professores deveriam de lançar mão dessa estratégia mais frequentemente (CAETANO; FALKENBACH, 2007).

\section{CONCLUSÕES}

Os resultados avaliados quantativamente $\mathrm{e}$ qualitativamente sobre o uso do vídeo na disciplina de anatomia animal permitiram concluir que este:

Constitui-se em uma ferramenta de suporte ao aprendizado divertida que promove satisfação pessoal, a integração do grupo, a divisão de tarefas e a responsabilidade.

Pode não ser fácil de desenvolver se houver sobrecarga de trabalhos, principalmente para os acadêmicos do sexo feminino.

Traz benefícios, estimulando o aprendizado através da experiência e por ser uma forma clara de apresentar o conteúdo.

A utilização do vídeo pode ser uma alternativa ao aprendizado, mas aspectos como disponibilidade de tempo do educador e dos acadêmicos, bem como suporte tecnológico e de material anatômico devem ser considerados para que haja um bom desenvolvimento do trabalho e sejam realizados vídeos boa qualidade.

\section{AGRADECIMENTOS}

Agradecemos às seguintes instituições e pessoas, por tornar possível a realização deste trabalho:

- A CAPES pela bolsa de pesquisador UAB

- Ao Núcleo de Tecnologia Educacional/ UFSM pelo apoio e seleção no edital 017/2012 NTE/UAB.

- Ao Núcleo de Sistemas de Informação (NUSI)/CCR na pessoa do Professor Claudio Baron Afonso Tiellet pela disponibilidade nos ensinamentos aos acadêmicos e pelas valiosas sugestões.

- Aos acadêmicos do Curso de Medicina Veterinária pela participação nesta pesquisa.

\section{REFERÊNCIAS BIBLIOGRÁFICAS}

ALMEIDA, D.S. et al. O vídeo na construção de uma educação do olhar. Perspectivas online. v. 3, n. 9, p. 153-174, 2009. Disponível em: http://www.perspectivasonline.com.br/revista/2009vol3n9/volume $\% 20$ 3(9)\%20artigo13.pdf Acesso: em mar. de 2013.

BRAVO, H.; INZUNZA, O. Evaluación de algunos programas computacionales en laensenãnza de anatomía y neuroanatomía de la facultad de Medicina de la Pontifícia Universidad Católica de Chile. Rev Chil Anat. v. 13, n. 1, p. 79-86, 1995.

CAETANO, S.V.N.; FALKEMBACH, G.A.M Youtube: uma opção para uso do vídeo na EAD. 10 p., 2007. Disponível em: http://www.cinted.ufrgs.br/ciclo9/ artigos/3aSaulo.pdf Acesso em: mar. de 2013.

CARNEIRO, V. L. Q. Linguagem audiovisual e objetivos pedagógicos: uma reflexão sobre os vídeos do Curso TV na Escola e os Desafios de Hoje. In: CONGRESSO INTERNACIONAL DE EDUCAÇÃO A DISTÂNCIA - SEED/MEC - ABED, 2001. Anais Eletrônicos... Brasília: ABED, 2001.

CORREIA, N.; CHAMBEL, T. Integração Multimedia em meios e ambientes aumentados nos contextos educativos e culturais. Arte e Ciência, n. 4, 22 p., 2004. Disponível em: http://www.multiciencia.unicamp.br/ artigos_02/a_02_.pdf. Acesso em: mar. de 2013.

CPQMVET. Dados do Perfil de Ingressantes e Acadêmicos - Parte 01 In: CPQMVet: Portfólio de Resultados do CPQMVet, n. 001, 2012. Disponível em: https:// docs.google.com/viewer?a $=$ v\&pid=sites\&srcid $=Z G V$ mYXVsdGRvbWFpbnxjcHFtdmV0dWZzbXxneDo0ODAyNjk5MTAxMDExNjE1 Acesso em: mar. de 2013.

DAVIS, B.T. Introduction to multimedia in museums. Disponível em: http://www.emuzeum.cz/admin/ clanky/files/280-introtomultimediamuseums.pdf 1996. Acesso em: jun. 2012.

FITZPATRICK, C. M. et al. Teaching anatomy with surgeons' tools: use of the laparoscope in clinical anatomy. Clin Anat, n. 14 p. 349-353, 2001

INFANTOSI, A. F. C.; KLEMT, A. Visualização 3D da dissecção crânio humano. Revista Brasileira de Engenharia Biomédica. v. 16, n. 1, p. 21-37, 2000.

KINNISON, T. et al. Teaching bovine abdominal anatomy: use of haptic simulator. Anat. Sci. Educ. v. 2, p. 
280-285, 2009. Disponível em: http://www.live.ac.uk/ documents/baillie/TeachingBovineAbdoAnatforwebsite_000.pdf Acesso em: mar. 2013

MORAN, J. M. O vídeo na sala de aula. Comunicação \& Educação. n. 2, pag. 27-35, 1995

Integração das Tecnologias na Educação. Desafios da televisão e do vídeo à escola. Secretaria de Educação a Distância, SEED. 2005

SOLOMON, G. Teach \& Learning. Sony Make Believe. Classroom video: tools and strategies in engage to students in learning. Disponível em: http://pro.sony.com/ bbsccms/ext/eblasts/edu/pdfs/class-room-ebook.pdf Acesso em: jun. 2013.

SANTORO, F. et al. Autoria cooperativa de crianças em sistemas hipermídias: relato de uma experiência. In: ED-MEDIA .1997, Calgary. Anais... Calgary, 1997.

SILVA, V. R., OLIVEIRA, E.M. As possibilidades do uso do vídeo como recurso de aprendizagem em salas de aula do 5 ano. Educação a Distância e Práticas Educativas Comunicacionais e Interculturais. n. 6, p. 93-103, 2010.

SILVA, K. C., SANTANA, O. A. Objetos de aprendizagem utilizados para o ensino da anatomia humana: uma revisão de literatura. 2012. Disponível em: http://www. abed.org.br/congresso2012/anais/123c.pdf Acesso em: mai. 2013

VALLE, L. R. L. D.; CRUZ, D. M. Reinventando a TV e o vídeo na escola: Uma experiência com a TV Escola e os professores da Rede Estadual de Ensino do Paraná. Athena. Revista Científica de Educação, v. 1, n. 1, jun./set. 2003.

VERRI, E.D. et al. Elaboração de vídeo-aula como ferramenta de complementar de aprendizagem dos discentes da área de saúde do Centro Universitário Claretiano de Batatais. Linguagem Acadêmica, v. 1, n. 1, p. 123-130, 2011.

YOSHIDA, M. et al. Locomoshow - uma ferramenta de apoio ao ensino da anatomia humana. 2003. Disponível em: http://www.sagha.com.br/locomoshow/ pdf/locomoshow.pdf Acesso em: jun. 2012. 